\section{Análise comparativa de classificações de causas evitáveis de morte em capitais brasileiras: 0 caso das doenças cerebrovasculares}

\author{
Daisy Maria Xavier de Abreu* \\ Eliane de Freitas Drumond ${ }^{\star *}$ \\ Elisabeth Barboza França*** \\ Lenice Harumi Ishitami ${ }^{\star \star * \star}$ \\ Deborah Carvalho Malta ${ }^{* * * *}$ \\ Carla Jorge Machado ${ }^{\star \star \star \star \star \star}$
}

\section{Introdução}

A mortalidade evitável caracteriza-se como uma abordagem que avança e qualifica as análises sobre variações temporais e regionais da mortalidade, ao identificar e mensurar as mortes devido a patologias evitáveis por atenção médica adequada e oportuna. Desde os estudos de Rutstein et al. (1976), várias propostas de classificação de causas de morte evitáveis foram desenvolvidas, partindo da premissa de que se pode impedir uma morte prematura evitando o surgimento da doença ou tratando-a adequadamente quando essa se apresenta (CHARLTON et al.,
1983; MACKENBACH et al., 1990; HOLLAND, 1991; TREUNIET, 1999). Nessa perspectiva, um indicador que considere o conceito de morte evitável é ferramenta sensível e útil na avaliação de sistemas de saúde (NOLTE; MCKEE, 2003; GÓMEZ-ARIAS et al., 2009).

As propostas de classificação de causas evitáveis contemplam dimensões relacionadas ao processo de desenvolvimento econômico-social, assim como ao conhecimento médico-científico consolidado e acessível à população. A revisão bibliográfica de Nolte e McKee (2004) sobre as classificações de causas evitáveis é elucidativa da evolução dos estudos produzidos sobre o tema, entre os quais se destaca a tipologia elaborada por Simonato et al. (1998), que classificaram as causas evitáveis segundo níveis de prevenção: primária, incluindo promoção à saúde; secundária, por meio de detecção e tratamento precoce, incluindo rastreamento; e terciária, envolvendo tratamento e reabilitação. Já a proposta de Tobias e Jackson (2001) introduz pesos por nível de prevenção para cada causa evitável. Com base nessa última classificação, Nolte e McKee (2004) ampliaram a seleção de causas evitáveis, incorporando elementos das tipologias de Charlton e colaboradores (1983) e Mackenbach (2000).

Nos países em desenvolvimento, particularmente na América Latina, a maior parte dos estudos de mortalidade evitável baseia-se na tipologia criada por Taucher (1978), que estabelece uma categorização de causas evitáveis, considerando aquelas passíveis de serem evitadas por meio de ações de saúde pública e melhorias nas condições de vida da população (SOLIS et

\footnotetext{
* Doutora em Saúde Pública, socióloga, pesquisadora do Núcleo de Estudos em Saúde Coletiva da Universidade Federal de Minas Gerais - UFMG.

** Doutora em Saúde Pública, médica, técnica da Secretaria Municipal de Saúde de Belo Horizonte, coordenadora do Sistema de Informações de Mortalidade no Município de Belo Horizonte.

*** Doutora em Saúde Pública, médica, professora do Programa de Pós-Graduação em Saúde Pública da Universidade Federal de Minas Gerais - UFMG.

**** Doutora em Saúde Pública, médica, técnica da Secretaria Municipal de Saúde de Belo Horizonte, Gerência de Epidemiologia e Informação.

${ }_{* \star \star \star \star}$ Doutora em Saúde Coletiva, médica, coordenadora geral de Doenças e Agravos não Transmissíveis, da Secretaria de Vigilância em Saúde do Ministério da Saúde.

${ }^{\star \star}$ Ph.D in Population Dynamics, professora do Centro de Desenvolvimento e Planejamento Regional - Cedeplar, da Universidade Federal de Minas Gerais - UFMG e do Programa de Pós-Graduação em Saúde Pública da Universidade Federal de Minas Gerais.
} 
al., 1982; PARPINELLI et al., 2000; NORIEGA BRAVO; ASTRAIN RODRIGUES, 2004).

No Brasil, no início da década de 1980 , a Fundação Sistema Estadual de Análise de Dados - Seade, de São Paulo, avançou na proposta de definição de critérios de evitabilidade para a mortalidade na infância, com base na tipologia de Taucher (1978). Foram criados grupos de causas evitáveis: por imunoprevenção; por adequado controle na gravidez; por adequada atenção ao parto; por ações de prevenção, diagnóstico e tratamento precoces; e por parcerias com outros setores (ORTIZ, 1983). Vários estudos sobre mortalidade evitável na população infantil no Brasil basearam-se nessa listagem (ORTIZ, 1983; HARTZ et al., 1996; MONTEIRO; SCHMITZ, 2004), que foi aplicada especialmente para a mortalidade neonatal (LEAL; SZWARCWALD, 1996; RIBEIRO; SILVA, 2000).

Em 2007, sob a coordenação da Secretaria de Vigilância em Saúde do Ministério da Saúde, foi publicada a primeira versão da Lista Brasileira de Causas Evitáveis (LBE), produto do trabalho de especialistas com o propósito de identificar as causas preveníveis, total ou parcialmente, por ações efetivas dos serviços de saúde disponíveis no Sistema Único de Saúde (MALTA et al., 2007).

As diferenças no perfil epidemiológico entre a população infantil e a adulta também devem ser introduzidas nas análises de tendências e variações da mortalidade evitável (MALTA; DUARTE, 2007). Partindo dessa consideração, a LBE propõe duas tipologias de causas evitáveis: uma para menores de cinco anos e outra para maiores de cinco até 75 anos de idade. Após a publicação da primeira versão da LBE e com base em discussões, análises e validação das causas selecionadas, recentemente foi proposta sua atualização para o grupo de menores de cinco anos, com enfoque especial no período neonatal (MALTA et al., 2010).

\section{Objetivo e justificativa do projeto de pesquisa}

Tendo em vista que a escolha das causas influencia a inferência sobre as análises de mortalidade evitável, deve-se proceder a uma permanente avaliação da seleção de causas evitáveis, em face do desenvolvimento e da introdução de novas tecnologias médicas (FRENCH; JONES, 2006). Além disso, a comparação de classificações de causas evitáveis evidencia que aquelas adotadas frequentemente variam em função do quadro epidemiológico local, dos avanços tecnológicos e também dos objetivos da lista empregada (GOMÉZ-ÁRIAS et al., 2009).

O quadro epidemiológico brasileiro recente tem se caracterizado pela redução das doenças infecciosas e parasitárias, que foram ultrapassadas pelas doenças cardiovasculares. Em anos recentes, estas últimas constituem a primeira causa de morte, seguidas pelas neoplasias e causas externas, compondo o grupo das doenças e agravos não-transmissíveis (BRASIL, 2007). Essa transição tem impactado a área de saúde pública no Brasil, tornando-se prioridade o desenvolvimento de estratégias para prevenção e controle destas patologias. A validação dos grupos de causas selecionadas como evitáveis ampliará as possibilidades de comparação do comportamento da mortalidade por essas causas entre municípios, regiões do país ou mesmo países.

Nesse contexto insere-se o projeto "Tendências da mortalidade por causas evitáveis em capitais brasileiras no período 1997 a 2006", que está sendo desenvolvido por pesquisadoras da Secretaria Municipal de Saúde de Belo Horizonte, do Departamento de Demografia/UFMG, do Nescon/ UFMG, do Programa de Pós-Graduação em Saúde Pública/UFMG, desde fevereiro de 2010 (número 15483 - Edital Universal Fapemig). O objetivo principal consiste em avaliar o comportamento da mortalidade por causas evitáveis selecionadas em capitais brasileiras, no decorrer de dez anos (19972006). A pesquisa foi aprovada pelo Comitê de Ética em Pesquisa da Universidade Federal de Minas Gerais, sob o protocolo número 143/09.

\section{Desenho do estudo}

O presente estudo direciona-se para causas evitáveis mais prevalentes na popu- 
lação adulta brasileira, a saber: doenças isquêmicas do coração (CID I20-I25); doenças cerebrovasculares (CID I61, I63.0-163.5, I63.8, I63.9, I64-I66); doenças hipertensivas (CID I10-I13); e diabetes mellitus (CID E10-E14).

O primeiro passo do estudo foi analisar a seleção de causas evitáveis com base na LBE. Observou-se que o grupo de doenças cerebrovasculares da LBE contemplou uma seleção diferente e menos inclusiva do que tem sido considerado por outras classificações de causas evitáveis. Portanto, para avaliar o escopo da seleção feita pela LBE, esta foi comparada com a lista proposta por Nolte e McKee (2004), que abrange toda a categoria de três caracteres referente às doenças cerebrovasculares (CID 10 I60 a l691), como também acontece em outras classificações de causas evitáveis (CHARLTON et al., 1983; HOLLAND, 1991), enquanto a LBE engloba parte da categoria (CID 10 I61; I630-I635; I638-I639; I64-I66²) (MALTA et al., 2007).

A partir dessa observação, foram selecionados os óbitos por doenças cerebrovasculares em residentes nas capitais brasileiras, no período de 2005 a 2007, segundo duas listas de causas evitáveis distintas. Optou-se por selecionar as 27 capitais devido à melhor qualidade da informação sobre mortalidade nesses municípios. A faixa etária estudada - maiores de cinco e menores de 75 anos de idade - é a proposta pela LBE.
Com o intuito de evitar flutuações aleatórias, os óbitos referem-se à média do triênio 2005-2007 para as capitais brasileiras.

\section{Resultados}

No total das capitais brasileiras, a classificação Nolte e McKee (2004) abrange cerca de $37 \%$ a mais de óbitos evitáveis por doenças cerebrovasculares do que a da LBE. Na Tabela 1, observam-se as diferenças relativas entre as duas listas, segundo sua localização nas regiões brasileiras. As capitais da Região Sul apresentam a maior diferença $(44,1 \%)$, seguidas por aquelas do Centro-Oeste $(42,6 \%)$, Sudeste $(36,9 \%)$, Nordeste $(35,5 \%)$ e Norte $(30,3 \%)$

Na Tabela 2 são apresentadas a mortalidade proporcional e as taxas de mortalidade por doenças cerebrovasculares nas capitais. Também em relação às taxas são observadas diferenças relativas importantes entre as duas listas em todas as capitais, com valores maiores na de Nolte e McKee (2004). As diferenças variaram de 16,3\%, em Belém, a 48,0\%, em Manaus.

Os óbitos por doenças cerebrovasculares, desagregados segundo os códigos da Lista Nolte, encontram-se na Tabela 3, sendo ressaltados em negrito aqueles não incluídos na LBE. Verifica-se que as diferenças maiores ocorrem em relação aos códigos I60.9, 167.8 e 169.4 .

\footnotetext{
${ }^{1}$ Capítulo IX - Doenças do aparelho circulatório: 160 a I69 - Doenças cerebrovasculares.

2 Capítulo IX - Doenças do aparelho circulatório: I61 - Hemorragia intracerebral; I630 - Infarto cerebral devido a trombose de artérias pré-cerebrais; I631 - Infarto cerebral devido a embolia de artérias pré-cerebrais; I632 - Infarto cerebral devido a oclusão ou estenose não especificadas de artérias pré-cerebrais; 1633 - Infarto cerebral devido a trombose de artérias cerebrais; 1634 - Infarto cerebral devido a embolia de artérias cerebrais; I635 - Infarto cerebral devido a oclusão ou estenose não especificadas de artérias cerebrais; 1638 - Outros infartos cerebrais; 1639 - Infarto cerebral não especificado; 164 - Acidente vascular cerebral, não especificado como hemorrágico ou isquêmico; I65 - Oclusão e estenose de artérias pré-cerebrais que não resultam em infarto cerebral; 166 - Oclusão e estenose de artérias cerebrais que não resultam em infarto cerebral.
} 
TABELA 1

Óbitos evitáveis por doenças cerebrovasculares (1), segundo as classificações de evitabilidade de Nolte e LBE Capitais brasileiras $-\mathbf{2 0 0 5 - 2 0 0 7}$

\begin{tabular}{|c|c|c|c|}
\hline Capitais & Nolte (2) & LBE (3) & $\begin{array}{c}\text { Diferença relativa (\%) } \\
{[(2)-(3)] /(2)}\end{array}$ \\
\hline Norte & 944 & 657 & 30,3 \\
\hline Porto Velho & 94 & 68 & 27,8 \\
\hline Rio Branco & 65 & 46 & 29,7 \\
\hline Manaus & 303 & 157 & 48,2 \\
\hline Boa Vista & 39 & 22 & 44,0 \\
\hline Belém & 375 & 313 & 16,4 \\
\hline Macapá & 41 & 32 & 21,8 \\
\hline Palmas & 27 & 20 & 28,0 \\
\hline Nordeste & 3.007 & 1.939 & 35,5 \\
\hline São Luís & 246 & 159 & 35,5 \\
\hline Teresina & 241 & 155 & 35,9 \\
\hline Fortaleza & 551 & 367 & 33,4 \\
\hline Natal & 140 & 84 & 39,9 \\
\hline João Pessoa & 194 & 135 & 30,3 \\
\hline Recife & 507 & 271 & 46,5 \\
\hline Maceió & 318 & 225 & 29,2 \\
\hline Aracaju & 116 & 69 & 41,0 \\
\hline Salvador & 693 & 475 & 31,5 \\
\hline Sudeste & 5.848 & 3.690 & 36,9 \\
\hline Belo Horizonte & 589 & 345 & 41,5 \\
\hline Vitória & 99 & 53 & 47,0 \\
\hline Rio de Janeiro & 2.217 & 1.445 & 34,8 \\
\hline São Paulo & 2.942 & 1.847 & 37,2 \\
\hline Sul & 984 & 550 & 44,1 \\
\hline Curitiba & 435 & 240 & 44,8 \\
\hline Florianópolis & 54 & 31 & 43,6 \\
\hline Porto Alegre & 495 & 280 & 43,5 \\
\hline Centro-Oeste & 1.125 & 646 & 42,6 \\
\hline Campo Grande & 217 & 127 & 41,5 \\
\hline Cuiabá & 137 & 85 & 38,1 \\
\hline Goiânia & 285 & 149 & 47,8 \\
\hline Brasília & 485 & 285 & 41,3 \\
\hline Total & 11.907 & 7.482 & 37,2 \\
\hline
\end{tabular}

Fonte: Datasus - SIM.

(1) Referem-se à faixa etária de maiores de cinco e menores de 75 anos.

(2) CID 10 - I60-I69.

(3) CID 10 - 161; I630-1635; 1638-1639; 164-166. 
TABELA 2

Óbitos por doenças cerebrovasculares, participação no total de óbitos e taxas de mortalidade (1), segundo as classificações de evitabilidade de Nolte e LBE Capitas brasileiras - 2005-2007

\begin{tabular}{|c|c|c|c|c|c|c|c|}
\hline \multirow{2}{*}{ Capitais } & \multirow{2}{*}{$\begin{array}{c}\text { Total de } \\
\text { óbitos }\end{array}$} & \multicolumn{3}{|c|}{ Lista Nolte (2) } & \multicolumn{3}{|c|}{ LBE (3) } \\
\hline & & N. abs. & $\%$ no total & Taxas (4) & N. abs. & $\%$ no total & Taxas (4) \\
\hline Porto Velho & 1.289 & 94 & 7,3 & 28,0 & 68 & 5,3 & 20,2 \\
\hline Rio Branco & 895 & 65 & 7,3 & 23,9 & 46 & 5,1 & 16,8 \\
\hline Manaus & 4.628 & 303 & 6,5 & 20,4 & 157 & 3,4 & 10,6 \\
\hline Boa Vista & 636 & 39 & 6,1 & 18,0 & 22 & 3,4 & 10,1 \\
\hline Belém & 4.273 & 375 & 8,8 & 29,4 & 313 & 7,3 & 24,6 \\
\hline Macapá & 747 & 41 & 5,5 & 13,1 & 32 & 4,3 & 10,2 \\
\hline Palmas & 345 & 27 & 7,9 & 14,1 & 20 & 5,7 & 10,1 \\
\hline São Luís & 2.724 & 246 & 9,0 & 27,6 & 159 & 5,8 & 17,8 \\
\hline Teresina & 2.328 & 241 & 10,4 & 33,8 & 155 & 6,6 & 21,7 \\
\hline Fortaleza & 7.369 & 551 & 7,5 & 25,6 & 367 & 5,0 & 17,1 \\
\hline Natal & 2.244 & 140 & 6,2 & 19,9 & 84 & 3,7 & 11,9 \\
\hline João Pessoa & 2.185 & 194 & 8,9 & 32,2 & 135 & 6,2 & 22,5 \\
\hline Recife & 6.239 & 507 & 8,1 & 37,4 & 271 & 4,3 & 20,0 \\
\hline Maceió & 3.494 & 318 & 9,1 & 39,0 & 225 & 6,4 & 27,6 \\
\hline Aracaju & 1.603 & 116 & 7,3 & 25,8 & 69 & 4,3 & 15,2 \\
\hline Salvador & 8.883 & 693 & 7,8 & 28,4 & 475 & 5,3 & 19,4 \\
\hline Belo Horizonte & 8.449 & 589 & 7,0 & 27,3 & 345 & 4,1 & 16,0 \\
\hline Vitória & 1.187 & 99 & 8,4 & 34,8 & 53 & 4,4 & 18,4 \\
\hline Rio de Janeiro & 29.164 & 2.217 & 7,6 & 40,6 & 1.445 & 5,0 & 26,5 \\
\hline São Paulo & 38.904 & 2.942 & 7,6 & 29,9 & 1.847 & 4,7 & 18,8 \\
\hline Curitiba & 6.246 & 435 & 7,0 & 27,0 & 240 & 3,8 & 14,9 \\
\hline Florianópolis & 1.156 & 54 & 4,7 & 14,7 & 31 & 2,7 & 8,3 \\
\hline Porto Alegre & 6.217 & 495 & 8,0 & 38,4 & 280 & 4,5 & 21,7 \\
\hline Campo Grande & 2.542 & 217 & 8,5 & 31,6 & 127 & 5,0 & 18,5 \\
\hline Cuiabá & 1.838 & 137 & 7,5 & 28,1 & 85 & 4,6 & 17,4 \\
\hline Goiânia & 4.281 & 285 & 6,7 & 25,9 & 149 & 3,5 & 13,5 \\
\hline Brasília & 6.320 & 485 & 7,7 & 22,8 & 285 & 4,5 & 13,4 \\
\hline Total & 156.187 & 11.907 & 7,6 & 30,0 & 7.482 & 4,8 & 18,9 \\
\hline
\end{tabular}

Fonte: Datasus - SIM.

(1) Referem-se à faixa etária de maiores de cinco e menores de 75 anos.

(2) CID $10-160-169$.

(3) CID 10 - I61; I630-I635; I638-I639; I64-I66.

(4) Por 100 mil habitantes. 
TABELA 3

Distribuição dos óbitos por doenças cerebrovasculares (1), segundo códigos da CID 10 constantes na Lista Nolte Capitais brasileiras - média anual 2005-2007

\begin{tabular}{|c|c|c|c|c|c|c|c|}
\hline \multirow{2}{*}{ CID10 4C Cap 09} & \multirow{2}{*}{$\begin{array}{c}\text { Norte } \\
\text { Capitais }\end{array}$} & \multirow{2}{*}{$\begin{array}{c}\text { Nordeste } \\
\text { Capitais }\end{array}$} & \multirow{2}{*}{$\begin{array}{l}\text { Sudeste } \\
\text { Capitais }\end{array}$} & \multirow{2}{*}{$\begin{array}{c}\text { Sul } \\
\text { Capitais }\end{array}$} & \multirow{2}{*}{$\begin{array}{l}\text { C-Oeste } \\
\text { Capitais }\end{array}$} & \multicolumn{2}{|c|}{ Brasil - Capitais } \\
\hline & & & & & & N. abs. & $\%$ \\
\hline I60.0 Hemorragia subarac. prov. sifão bifurc. carotid. & 0 & 0 & 1 & 0 & 0 & 2 & 0,0 \\
\hline I60.1 Hemorragia subarac. prov. artéria cerebr. média & 0 & 0 & 6 & 2 & 0 & 8 & 0,1 \\
\hline I60.2 Hemorragia subarac. prov. artéria comunic. ant. & 0 & 0 & 3 & 0 & 1 & 5 & 0,0 \\
\hline I60.3 Hemorragia subarac. prov. artéria comunic. post. & 0 & 0 & 1 & 0 & 1 & 2 & 0,0 \\
\hline I60.4 Hemorragia subarac. prov. artéria basilar & 0 & 1 & 1 & 1 & 0 & 2 & 0,0 \\
\hline I60.5 Hemorragia subarac. prov. artéria vertebral & 0 & 0 & 0 & 0 & 0 & 0 & 0,0 \\
\hline I60.6 Hemorragia subarac. prov. outr. artér. intracran. & 0 & 1 & 1 & 1 & 0 & 2 & 0,0 \\
\hline I60.7 Hemorragia subarac. prov. artéria intracran. NE & 1 & 5 & 7 & 2 & 1 & 15 & 0,1 \\
\hline I60.8 Outras hemorragias subaracnoides & 1 & 6 & 92 & 3 & 1 & 104 & 0,9 \\
\hline I60.9 Hemorragia subaracnoide NE & 68 & 201 & 575 & 115 & 106 & 1.065 & 9,0 \\
\hline I61.0 Hemorragia intracerebral hemisf. subcortical & 0 & 0 & 1 & 0 & 0 & 2 & 0,0 \\
\hline I61.1 Hemorragia intracerebral hemisférica cortical & 0 & 0 & 0 & 0 & 0 & 1 & 0,0 \\
\hline I61.2 Hemorragia intracerebral hemisférica NE & 0 & 1 & 0 & 2 & 0 & 3 & 0,0 \\
\hline I61.3 Hemorragia intracerebral do tronco cerebral & 1 & 2 & 19 & 4 & 3 & 28 & 0,2 \\
\hline I61.4 Hemorragia intracerebral cerebelar & 0 & 3 & 11 & 3 & 2 & 18 & 0,2 \\
\hline I61.5 Hemorragia intracerebral intraventricular & 1 & 4 & 14 & 3 & 2 & 25 & 0,2 \\
\hline 161.6 Hemorragia intracerebral de mult. localiz. & 0 & 0 & 2 & 1 & 0 & 3 & 0,0 \\
\hline 161.8 Outras hemorragias intracerebrais & 1 & 1 & 4 & 9 & 2 & 17 & 0,1 \\
\hline I61.9 Hemorragia intracerebral NE & 216 & 808 & 1.418 & 229 & 309 & 2.979 & 25,2 \\
\hline I62.0 Hemorragia subdural & 4 & 15 & 41 & 9 & 5 & 73 & 0,6 \\
\hline I62.1 Hemorragia extradural não-traum. & 0 & 1 & 0 & 0 & 0 & 1 & 0,0 \\
\hline I62.9 Hemorragia intracraniana NE & 6 & 8 & 27 & 5 & 2 & 48 & 0,4 \\
\hline I63.0 Infarto cerebr. dev. tromb. artérias pré-cerebr. & 0 & 1 & 3 & 1 & 0 & 6 & 0,0 \\
\hline I63.1 Infarto cerebr. dev. embol. artérias pré-cerebr. & 0 & 1 & 2 & 0 & 0 & 3 & 0,0 \\
\hline I63.2 Inf. cer. dev. oclusão esten. NE art. pré-cerebr. & 0 & 3 & 6 & 2 & 1 & 12 & 0,1 \\
\hline I63.3 Infarto cerebr. dev. trombose artérias cerebr. & 1 & 1 & 2 & 1 & 1 & 6 & 0,1 \\
\hline I63.4 Infarto cerebral dev. embolia artérias cerebr. & 0 & 1 & 0 & 1 & 0 & 2 & 0,0 \\
\hline I63.5 Inf. cer. dev. oclusão estenose NE art. cerebr. & 0 & 0 & 2 & 3 & 0 & 4 & 0,0 \\
\hline I63.6 Inf. cerebr. dev. tromb. ven. cerebr. não-piogenic. & 0 & 0 & 0 & 0 & 0 & 0 & 0,0 \\
\hline I63.8 Outros infartos cerebrais & 1 & 3 & 4 & 3 & 0 & 11 & 0,1 \\
\hline I63.9 Infarto cerebral NE & 33 & 21 & 428 & 45 & 10 & 537 & 4,5 \\
\hline 164 Acid. vasc. cerebr. NE como hemorrag. isquêmico & 335 & 1.089 & 1.775 & 242 & 315 & 3.756 & 31,8 \\
\hline I67.0 Dissecção de artérias cerebrais s/ruptura & 0 & 0 & 0 & 0 & 0 & 1 & 0,0 \\
\hline I67.1 Aneurisma cerebral não-roto & 10 & 24 & 57 & 4 & 18 & 113 & 1,0 \\
\hline I67.2 Aterosclerose cerebral & 0 & 21 & 20 & 1 & 1 & 43 & 0,4 \\
\hline I67.3 Leucoencefalopatia vascular progressiva & 0 & 0 & 0 & 0 & 0 & 0 & 0,0 \\
\hline I67.4 Encefalopatia hipertensiva & 9 & 32 & 33 & 2 & 6 & 83 & 0,7 \\
\hline I67.5 Doença de Moyamoya & 0 & 0 & 0 & 0 & 0 & 0 & 0,0 \\
\hline I67.6 Trombose não-piogenica sist. venoso intracran. & 0 & 0 & 0 & 0 & 0 & 0 & 0,0 \\
\hline I67.7 Arterite cerebral NCOP & 0 & 0 & 1 & 1 & 0 & 2 & 0,0 \\
\hline I67.8 Outras doenças cerebrovasculares espec. & 61 & 400 & 585 & 126 & 194 & 1.367 & 11,6 \\
\hline I67.9 Doença cerebrovascular NE & 3 & 9 & 16 & 5 & 3 & 36 & 0,3 \\
\hline I69.0 Sequelas de hemorragia subaranoidea & 5 & 2 & 5 & 4 & 1 & 17 & 0,1 \\
\hline I69.1 Sequelas de hemorragia intracerebral & 0 & 1 & 9 & 5 & 4 & 20 & 0,2 \\
\hline I69.2 Sequelas outr. hemorrag. intracran. não traum. & 0 & 0 & 5 & 1 & 0 & 6 & 0,0 \\
\hline I69.3 Sequelas de infarto cerebral & 0 & 1 & 48 & 19 & 4 & 72 & 0,6 \\
\hline I69.4 Sequelas acid. vasc. cerebr. NE c/hemorr. isquêm. & 83 & 320 & 602 & 120 & 118 & 1.244 & 10,5 \\
\hline I69.8 Sequelas outr. doenças cerebrovasculares e NE & 7 & 19 & 21 & 8 & 13 & 68 & 0,6 \\
\hline Total & 850 & 3.007 & 5.848 & 984 & 1.125 & 11.813 & 100,0 \\
\hline
\end{tabular}

Fonte: Datasus - SIM.

(1) Referem-se à faixa etária de maiores de cinco e menores de 75 anos.

Nota: Os códigos destacados em negrito não constam na LBE.

Foram excluídas aquelas causas nas quais não foi registrado nenhum óbito. 


\section{Discussão dos resultados preliminares e perspectivas}

O estudo atual buscou comparar os óbitos por doenças cerebrovasculares em residentes nas capitais brasileiras, no período de 2005 a 2007, segundo duas listas de causas evitáveis distintas, na faixa etária de maiores de cinco e menores de 75 anos. Comparando-se as duas propostas de mortes por causas evitáveis, encontrou-se uma diferença de $37 \%$ de óbitos no triênio, sendo menor o número de mortes segundo a Lista Brasileira de Causas Evitáveis.

As diferenças encontradas devem ser mais bem exploradas, o que será realizado na próxima etapa do trabalho. Em geral, as listas de causas evitáveis variam em função de condições locais, padrão epidemiológico, incorporação de novas tecnologias, o que possibilita incluir causas antes não consideradas evitáveis. A LBE foi criada com o objetivo de expressar o padrão epidemiológico no Brasil, onde predominam as DCNT, mas ainda com dupla carga de doenças, além de refletir as condições assistenciais do país, o nível de acesso a tecnologias, em especial aquelas disponíveis no Sistema Único de Saúde. Já a lista de Nolte e Mckee reflete a incorporação dos avanços nos países europeus e um quadro epidemiológico em que a dupla carga de doenças já foi superada. Estas diferenças podem inicialmente explicar os achados deste estudo, mas novas validações tornamse necessárias.

Em face dos resultados encontrados, considerou-se importante proceder a uma validação da codificação de causas relacionadas aos códigos da CID 10 utilizados

\section{Referências}

BRASIL. Ministério da Saúde. Secretaria de Vigilância em Saúde. Departamento de Análise de Situação em Saúde. Saúde Brasil 2007: uma análise da situação de saúde. Brasília: Ministério da Saúde, 2007.

CHARLTON, J. R. H.; SILVER, R.; HARTLEY, R. M.; HOLLAND, W. W. Geographical variation in mortality from conditions amenable to para codificar as doenças cerebrovasculares. Para tal, solicitou-se a 14 codificadores capacitados - gestores responsáveis pelo SIM nas capitais e Estados das Regiões Sul, Sudeste e Centro-Oeste - a codificação da mesma declaração de óbito, na qual o acidente vascular cerebral isquêmico estava incluído entre as causas de morte declaradas. A codificação foi feita por cada profissional de forma cega e independente. Buscava-se, assim, verificar semelhanças ou diferenças nos códigos utilizados para definir a causa básica em DO com a mesma sequência de causas de morte. $O$ código 167.8 (outras doenças cerebrovasculares especificadas) não incluído na LBE, foi utilizado por nove codificadores $(64,3 \%)$, o 163.9 (infarto cerebral não especificado) por três, o 161.9 (hemorragia intracerebral não especificada) e o I64 (acidente vascular cerebral com hemorragia isquêmico) por um codificador cada.

Conforme destaca Santo (2000), a avaliação crítica da codificação das causas de morte deve ser uma tarefa contínua e permanente, de modo a identificar problemas de codificação, que podem ser prevenidos por meio de um programa de controle de qualidade da codificação das causas de morte no Sistema de Informações sobre Mortalidade no Brasil.

Esses resultados reforçam a necessidade de uma contínua revisão e atualização das listas de causas evitáveis, de modo que as mesmas possam servir como um importante instrumento na avaliação da atenção à saúde prestada. Outros desdobramentos da pesquisa serão direcionados para entender as diferenças encontradas entre os municípios brasileiros.

medical intervention in England and Wales. The Lancet, v. 26, p. 691-696, 1983.

FRENCH, K. M.; JONES, K. Impact of definition on the study of avoidable mortality: geographical trends in British deaths 1981-1998 using Charlton and Holland's definitions. Soc. Sci. Med., v. 62, n. 6, p. $1.443-56,2006$. 
GOMÉZ-ARIAS, R. D.; BONMATÍ, A. N.; PEREYRA-ZAMORA, P.; ARIAS-VALENCIA, S.; RODRÍGUEZ-OSPINA, F. L.; AGUIRRE, D. C. Diseño y análisis comparativo de un inventario de indicadores de mortalidad evitable adaptado a las condiciones sanitarias de Colombia. Rev. Panam. Salud Publica, v. 26, n. 5, p. 385-397, 2009.

HARTZ, Z. M. A.; CHAMPAGNE, F.; CONTRANDIOPOULOS, A.; LEAL, M. C. "Avoidable" infant mortality in two cities of Northeastern Brazil: quality indicator of the local health system. Journal of Public Health, v. 30, n. 4, p. 310-8, 1996.

HOLLAND, W. W.; EC WORKING GROUP ON HEALTH SERVICES AND "AVOIDABLE DEATHS". European community atlas of avoidable death. 2nd ed. Oxford: Oxford University Press, 1991 (Oxford medical publications, Commission of the European Communities Health Services Research Series n. 6, 9).

LEAL, M. C.; SZWARCWALD, C. L. Evolução da mortalidade neonatal no Estado do Rio de Janeiro, Brasil (1979-1993): análise por causa segundo grupo de idade e região de residência. Cad. Saúde Pública, v. 12, n. 2 , p. 243-252, 1996.

MACKENBACH, J. P. How important have medical advances been? In: SUSSEX, J. (Ed.). Improving population health in industralised countries. London: Office of Health Economics, 2000.

MACKENBACH, J. P.; BOUVIER-COLLE, M.H.; JOUGLA, E. "Avoidable" mortality and health services: a review of aggregate data studies. Journal of Epidemiology and Community Health, v. 44, p. 106-111, 1990.

MALTA, D. C.; DUARTE, E. C. Causas de morte evitáveis por ações efetivas dos serviços de saúde: uma revisão da literatura. Ciência e Saúde Coletiva, v. 12, n. 3, p. 765-776, 2007.

MALTA, D. C.; DUARTE, E. C.; ALMEIDA, M. F.; DIAS, M. A. S.; MORAIS NETO, O. L.; MOURA, L.; FERRAZ, W.; SOUZA, M. F. M. Lista de causas de mortes evitáveis por intervenções do Sistema Único de Saúde do Brasil. Epidemiol. Serv. Saúde, v. 16, n. 4, p. 233-244, 2007.
MALTA, D. C.; SARDINHA, L. M. V.; MOURA, L.; LANSKY, S.; LEAL, M. C.; SZARCWALD, C. L.; FRANÇA, E.; ALMEIDA, M.F.; DUARTE, E. C. Atualização da lista de causas de mortes evitáveis por intervenções do Sistema Único de Saúde do Brasil. Epidemiol. Serv. Saúde v. 19, n. 2, p. 173-176, 2010.

MONTEIRO, R. A.; SCHMITZ, B. A. S. Principais causas básicas da mortalidade infantil no Distrito Federal, Brasil: 1990-2000. Rev. Bras. Saúde Matern. Infant., v. 4, n. 4, p. 413-421, 2004.

NOLTE, E.; MCKEE, M. Measuring the health of nations: analysis of mortality amenable to health care. British Medical Journal, v. 327, n. 15, p. 1-5, 2003.

Does health care save lives? Avoidable mortality revisited. London: Nuffield Trust; 2004.

NORIEGA BRAVO, V. M.; ASTRAÍN RODRÍGUEZ, M. E. Avoidable mortality according to the living conditions of adults in La Lisa municipality, 1996-1998. Rev. Cubana de Salud Pública, v. 30 , n. 4, p.1-9, 2004.

ORTIZ, L. P. Evolução da mortalidade infantil no estado de São Paulo segundo sexo, idade e causas de morte. Informe Demográfico, n. 8, p. 93-135, 1983.

PARPINELLI, M.A.; FAÚNDES A.; CECATTI, J. G.; PEREIRA, B. G.; PASSINI JÚNIOR, R.; AMARAL, E. Analysis of avoidable mortality among women in reprodutive age. Rev. Bras. Ginecol. Obstet, v. 22, n.9, p.579-584. 2000.

SILVA, A. A. M. Tendência da mortalidade neonatal em São Luís, Maranhão, Brasil, de 1979 a 1996. Cad Saúde Pública, v. 16, n. 2, p. 429-436, 2000.

RUTSTEIN, D. D.; BERENBERG, W.; CHALMERS, T. C.; CHILD, C. G.; FISHMAN, A. P.; PERRIN, E. B. Measuring the quality of medical care. A clinical method. N. Engl. J. Med., v. 294, n. 11, p. 582-8, 1976.

SANTO, A. H. Avaliação da qualidade de codificação das causas de morte no estado de São Paulo, Brasil. Inf. Epidemiol. SUS, v. 9, n. 3, p. $189-198,2000$. 
SIMONATO, L.; BALLARD, T.; BELLINI, P.; WINKELMANN, R. Avoidable mortality in Europe 1955 - 1994: a plea for prevention. JECH, v. 52, n. 10, p. 624-630, 1998.

SOLIS, F.; CASTILLO, B.; MARDONES, G. Child mortality grouped by avoidable causes in the 27 health services of the country (Chile, 1979). Rev. Méd. Chile, v. 110, n.4, p. 383-90, 1982.

TAUCHER, E. La mortalidad en Chile desde 1955 a 1975; tendencias y causas. Notas de Población, v. 6, n. 18, p. 113-142, 1978.
TOBIAS, M.; JACKSON, G. Avoidable mortality in New Zealand, 1981-97. Aust N Z J Public Health, n. 25, p. 12-20, 2001.

TREURNIET, H. F.; LOOMAN, C. W. N.; MAAS, P. J.; MACKENBACH, J. P. Variations in "avoidable" mortality: a reflection of variations in incidence? International Journal of Epidemiology, n. 28, p. 225232, 1999.

Recebido para publicação em 15/08/2010 Aceito para publicação em 29/08/2010 